\title{
External Force Estimation of Pneumatic Soft Actuator with Built-in Displacement Sensor
}

\author{
Osamu Azami, ${ }^{1 *}$ Toshihiro Kawase, ${ }^{1,2}$ Tetsuro Miyazaki, ${ }^{3}$ \\ Takahiro Kanno, ${ }^{4}$ and Kenji Kawashima ${ }^{3}$ \\ ${ }^{1}$ Tokyo Medical and Dental University, 2-3-10, Kanda Surugadai, Chiyoda-ku, Tokyo 101-0062, Japan \\ ${ }^{2}$ Tokyo Institute of Technology, 4259, Nagatsuta-cho, Midori-ku, Yokohama 226-8503, Japan \\ ${ }^{3}$ Tokyo University, 7-3-1, Hongo, Bunkyo-ku, Tokyo 113-8656, Japan \\ ${ }^{4}$ Riverfield Inc., 20, Samoncho, Shinjuku-ku, Tokyo 160-0017, Japan
}

(Received October 30, 2020; accepted January 14, 2021)

Keywords: pneumatic, soft actuator, built-in sensor, force estimation, displacement estimation

We have proposed an extension-type pneumatic soft actuator made of a silicone tube covered by a metal spring. The spring is used to reinforce the outer periphery and to estimate the extension of the actuator from its change in inductance. However, the repeatability of the measurement needs to be improved since neither side of the spring is firmly fixed to the silicone tube. In this study, we newly designed and developed a soft actuator to improve the repeatability of the measurement. Then, we estimated the external force applied to the actuator using the supply pressure and the estimated displacement of the spring. In our experiments, we used an external force sensor, and its measured value was compared with the estimated force value. We confirmed the effectiveness of the force estimation by performing experiments with a lowfrequency sinusoidal input of $0.1 \mathrm{~Hz}$.

\section{Introduction}

Soft actuators are being actively studied since they are safe in contact with humans. ${ }^{(1)}$ Soft actuators are applied in the industrial ${ }^{(2,3)}$ and medical fields. ${ }^{(4)}$ For example, they are used in rehabilitation devices ${ }^{(5)}$ and surgical lamp operating devices. ${ }^{(6)}$ Soft actuators are also an important element in soft robots. Various types of elongating actuators have also been proposed. ${ }^{(7-10)}$ Hawkes developed a fiber-reinforced soft actuator with only one chamber. ${ }^{(11)}$ The maximum stroke of the actuator was about $300 \%$ of the natural length and was larger than that of conventional straight pneumatic cylinders. Fiber-reinforced actuators have high pressure resistance so they are used in various applications. ${ }^{(12)}$

In addition, soft actuators with buried sensors have been proposed, as well as a soft actuator incorporating a capacitive sensor. ${ }^{(13)}$ Not only electronic components but also conductive liquids and rubber can be used for soft actuators. Nguyen developed a conductive solid rubber buried actuator and measured its displacement from the change in electric resistance of the buried rubber. ${ }^{(14)}$ Actuators in which conductive ink ${ }^{(15)}$ or liquid $^{(16-20)}$ metal is encapsulated in a surface channel have also been proposed. We proposed a soft actuator with a built-in strain

*Corresponding author: e-mail: osamu_6464@yahoo.co.jp

https://doi.org/10.18494/SAM.2021.3152 
gauge to measure the displacement of the actuator. The opening angle of a forceps grasper was measured by the actuator. ${ }^{(21)}$ However, such an actuator with a built-in sensor ${ }^{(22-25)}$ requires a process for burying components during the manufacture, and the material of the sensor is often special or expensive. Therefore, we proposed a pneumatic soft actuator that enables a cover spring to be used as a sensor. ${ }^{(26)}$ This actuator consisted of a silicone tube and a metal spring covering the tube. The spring suppresses the radial expansion of the silicone tube and ensures the axial extension of the tube when it is charged with air. We measured the actuator displacement using the change in inductance of the metal spring. We confirmed the effectiveness of the displacement measurement through experiments. However, the repeatability of the measurement needed to be improved since neither side of the spring was firmly fixed to the silicone tube.

In this study, we newly designed and developed a soft actuator to fix the spring and the silicone tube to improve the repeatability of the displacement measurement. Then, we proposed a method of estimating the force applied to the soft actuator from the displacement and the pressure in the actuator. In the experiments, we used an external force sensor, and its measured value was compared with the estimated force value.

In Sect. 2, we give an outline of our developed soft actuator and its displacement estimation method. Section 3 describes the principle of the force estimation method and Sect. 4 describes the manufacturing process of the newly designed actuator and molds. Then, experiments performed to confirm the validity of the proposed method are reported in Sect. 5. Finally, we give conclusions in Sect. 6.

\section{Soft Actuator with Built-in Displacement Sensor}

We first describe the soft actuator with the built-in displacement sensor proposed in our previous work $^{(26)}$ and the problem to be solved.

\subsection{Structure of the soft actuator}

The developed soft actuator performs linear motion when supplied with compressed air (Fig. 1). A soft tube balloon made of silicone is covered by a metal spring. By supplying compressed air,

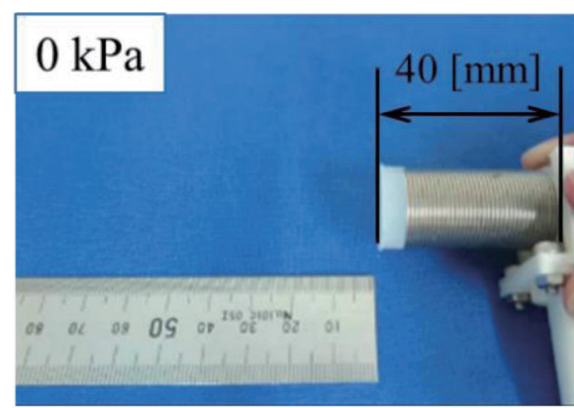

(a)

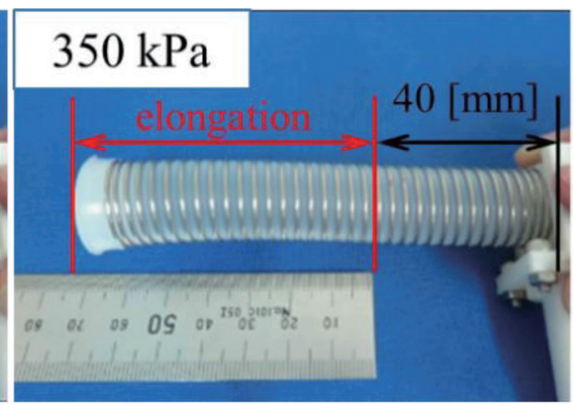

(b)

Fig. 1. (Color online) Deformation of the soft actuator. (a) $0 \mathrm{kPa}$ and (b) $350 \mathrm{kPa}$. 
the soft actuator suppresses radial expansion and undergoes axial extension. The metal spring is used to restrain the radial expansion during pressurization and to measure the displacement from the change in inductance with the length. The actuator weighs only $30 \mathrm{~g}$ and is lightweight. The actuator extends to about 2.5 times its initial length when a pressure of $350 \mathrm{kPa}$ is applied. Note that a slight error in the manufacturing process causes unintended small bending motion of the actuator as shown in Fig. 1. In the experiment, the bending is suppressed by using a pipe with an inner diameter larger than the outer diameter of the actuator so as not to hinder the linear motion.

\subsection{Principle of the displacement measurement}

The principle of displacement measurement using a spring is next briefly explained. The deformation of the actuator changes the length of the spring, and the inductance of the spring changes accordingly. The inductance of the spring is expressed by ${ }^{(27)}$

$$
L=\frac{K_{N} \mu \pi r^{2} N^{2}}{l}
$$

where $L[\mathrm{H}]$ is the inductance of the spring, $\mu[\mathrm{H} / \mathrm{m}]$ is the magnetic permeability, $N$ is the winding number, $r[\mathrm{~m}]$ is the radius of the spring, $l[\mathrm{~m}]$ is the length of the spring, and $K_{N}$ is the Nagaoka coefficient. $K_{N}$ can be represented as a function of $l$ and $r^{(28)}$ Assuming the radius of the spring does not change, $K_{N}$ is a function $f(l)$ that is only affected by the length. The frequency $f_{o}[\mathrm{~Hz}]$ of the spring connected to the circuit for inductance measurement is expressed by

$$
f_{o}=\frac{1}{2 \pi \sqrt{L C S}}
$$

where $C s[F]$ is the total capacitive of two capacitors. Figure 2 shows the circuit used for inductance measurement and a photograph taken when the proposed actuator is connected

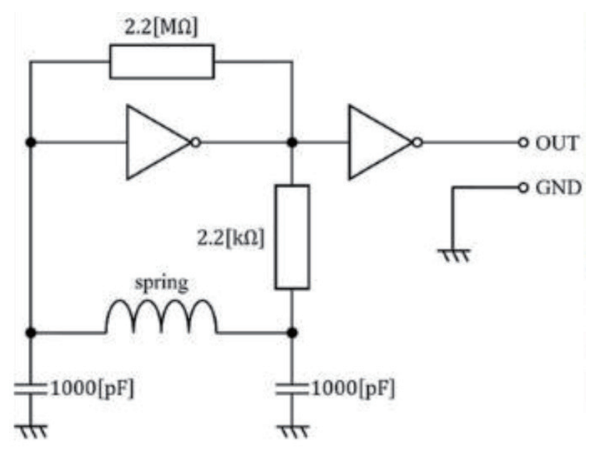

(a)

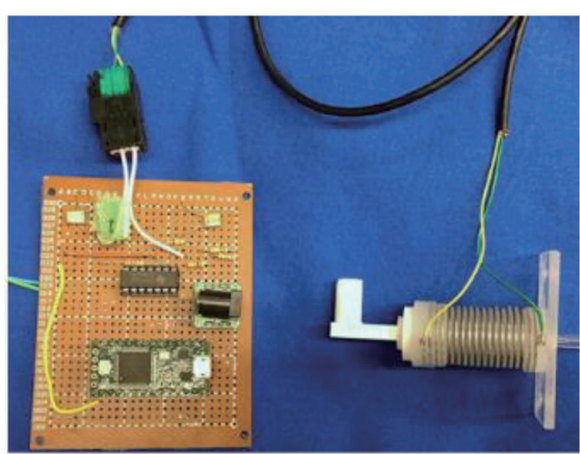

(b)

Fig. 2. (Color online) Circuit and actuator. (a) Circuit for inductance measurement. (b) Circuit connected to the proposed actuator. 
to the circuit. We use a spring inductance measurement circuit that uses a Pierce oscillator circuit. Both ends of the metal spring are connected to the inductance measurement circuit. By substituting Eq. (1) and $K_{N}=f(l)$ into Eq. (2), the following expression is derived:

$$
f_{\mathrm{o}}=\frac{1}{2 \pi \sqrt{\frac{\mu \pi r^{2} N^{2} f(l)}{l} C s}},
$$

where $\mu, N$, and $C s$ are constants. Therefore, if $r$ is constant, the oscillation frequency can be represented by a function that depends only on the length of the spring. Figure 3 shows the relationship between the actuator displacement and the oscillation frequency. Since the frequency is uniquely determined by the displacement, we can measure the displacement by measuring the oscillation frequency of the spring.

Using the proposed method, we controlled the actuator displacement without an additional external sensor such as a wire encoder. However, the spring covering the actuator was not fixed to the silicone tube and the position of the spring slightly shifted each time the actuator expanded and contracted. Therefore, the oscillation frequency changed slightly after several motions even when the elongation of the soft actuator was the same. It is therefore necessary to improve the repeatability of the displacement measurement. It is also necessary to measure the force acting on the soft actuator and desirable to apply it to robots that collaborate with humans.

\section{Force Estimation}

A pneumatic soft actuator has high backdrivability. It has been verified that the effective cross-sectional area of the soft actuator depends on the outer diameter of the silicone part. ${ }^{(29)}$ The force generated by the actuator can be calculated by multiplying the outer diameter by the supply pressure. Then, the force acting on the actuator can be calculated as

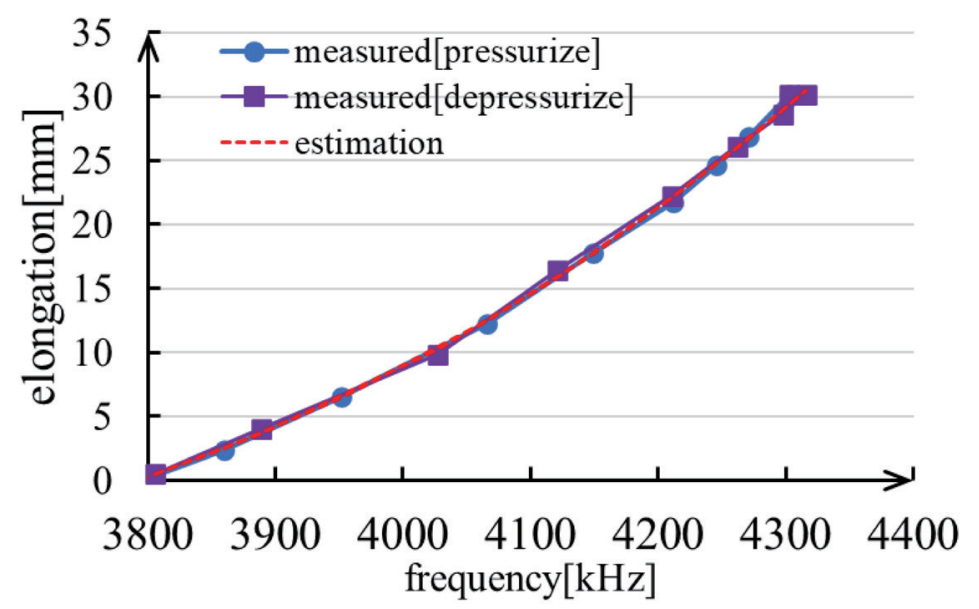

Fig. 3. (Color online) Relationship between oscillation frequency and actuator elongation. 


$$
F_{\text {est }}=A P-F\left(L_{x}\right)
$$

where $A$ is the effective cross-sectional area of the actuator, $F_{\text {est }}$ is the force applied to the actuator, $F\left(L_{x}\right)$ is the spring force of the actuator, and $L_{x} \mathrm{~mm}$ is the measured displacement of the actuator. Through preliminary experiments, the effective area of the actuator was measured as $2.409 \times 10^{-4} \mathrm{~m}^{2}$ and the radius was calculated as $17.51 \mathrm{~mm}$.

Then, we determined the spring force characteristics of the actuator. We assumed that the actuator is a spring with nonlinear characteristics. The relationship between the actuator driving force and the displacement obtained from the static characteristic experiment was approximated by a polynomial. Figure 4 shows the relationship between the elongation and force of the actuator, where the blue line and circles show the relationship between the driving force and elongation in the pressurization process, the purple line and squares show the relationship in the depressurization process, and the red dashed line shows the approximate curve. The actuator driving force was calculated from the supply pressure and the effective cross-sectional area of the actuator. As shown in Fig. 4, the proposed actuator has hysteresis. We consider that the hysteresis is caused by the flexibility and viscosity of silicone, the material of the actuator. We obtained the following approximate polynomial for estimating the force using the least-squares method:

$$
F\left(L_{x}\right) \cong 8.843 \times 10^{-3} L_{x}^{3}-3.710 \times 10^{-1} L_{x}{ }^{2}+8.645 L_{x}
$$

Equation (5) is an experimentally derived polynomial. Considering the units of the left and right terms, the units of the coefficients are $\mathrm{N} / \mathrm{m}^{3}, \mathrm{~N} / \mathrm{m}^{2}$, and $\mathrm{N} / \mathrm{m}$ for the first three terms on the right-hand side, respectively. Equation (4) suggests that the displacement of the soft actuator directly affects the estimation accuracy of the acting force. Therefore, to estimate the displacement with higher repeatability, we newly designed and manufactured an actuator in which part of the spring is fixed to the silicone tube.

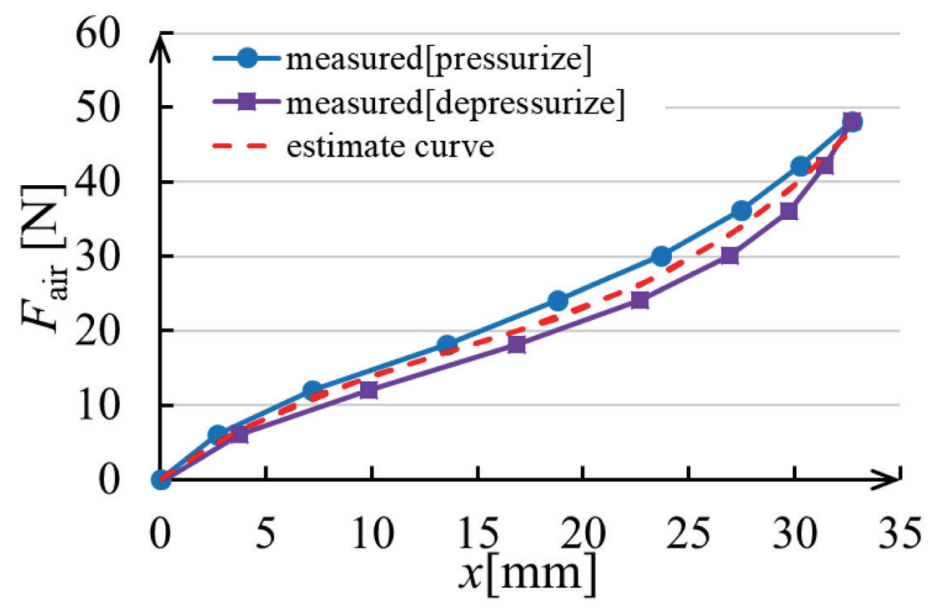

Fig. 4. (Color online) Nonlinear spring constant of actuator. 


\section{Newly Designed Actuator}

In the conventional fabrication process, ${ }^{(26)}$ the metal spring is covered after manufacturing a stepped silicone tube. It is difficult to fix a metal spring to a silicone tube by the fabrication process. Therefore, we proposed a fabrication procedure in which both ends of the spring are embedded and fixed in a silicone tube.

\subsection{New molds and fabrication process}

The new molds used to fabricate the soft actuator are shown in Fig. 5 and a detailed description of each mold is given in Table 1. We used the 3D printer Ultimaker 3 to make the molds, whose material is polylactic acid (PLA). The fabrication process of the actuator is shown in Fig. 6. First, the spring and mold No.3 are placed in mold No.1. The spring has soldered lead wires on both ends. Next, liquid silicone is poured into mold No.1 and mold No. 2 is placed on it. After removing the bubbles using a vacuum pump, mold No.4 is placed on mold No.2. The silicone is left to cure at $23{ }^{\circ} \mathrm{C}$ for $16 \mathrm{~h}$ in a constant-temperature bath. After curing, the silicone tube is removed from the molds and the opening at the end is closed with silicone. Finally, an

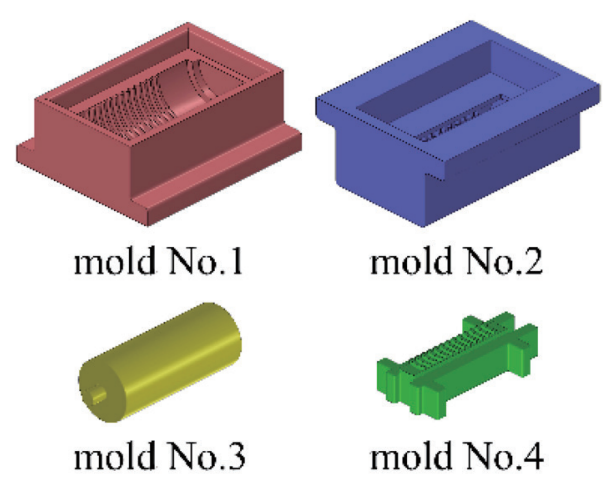

Fig. 5. (Color online) Four molds used to fabricate the soft actuator.

Table 1

Detailed description of each mold.

\begin{tabular}{|c|c|}
\hline Mold No.1 & $\begin{array}{l}\text { Bottom part fits spring and mold No.3 and is combined with mold No.2. } \\
\text { Curved spiral groove holds measuring spring. } \\
\text { Spiral groove fixes shape of spring and prevents silicone } \\
\text { entering gaps between windings. } \\
\text { No groove at both ends of interior. } \\
\text { Mold interior is cylindrical with same diameter as outer diameter of spring. }\end{array}$ \\
\hline Mold No.2 & $\begin{array}{l}\text { Lid on mold No.1. } \\
\text { Curved spiral groove is connected to groove of mold No.1. } \\
\text { Hole at top for air bubbles to escape. }\end{array}$ \\
\hline Mold No.3 & $\begin{array}{l}\text { Shaft that supports inner diameter of silicone tube. } \\
\text { Shape of stepped round bar for hole of air tube. }\end{array}$ \\
\hline Mold No.4 & $\begin{array}{l}\text { Lid to close hole of mold No.2. Gap for lead wires to escape. } \\
\text { Curved spiral groove is connected to groove of mold No.2. }\end{array}$ \\
\hline
\end{tabular}




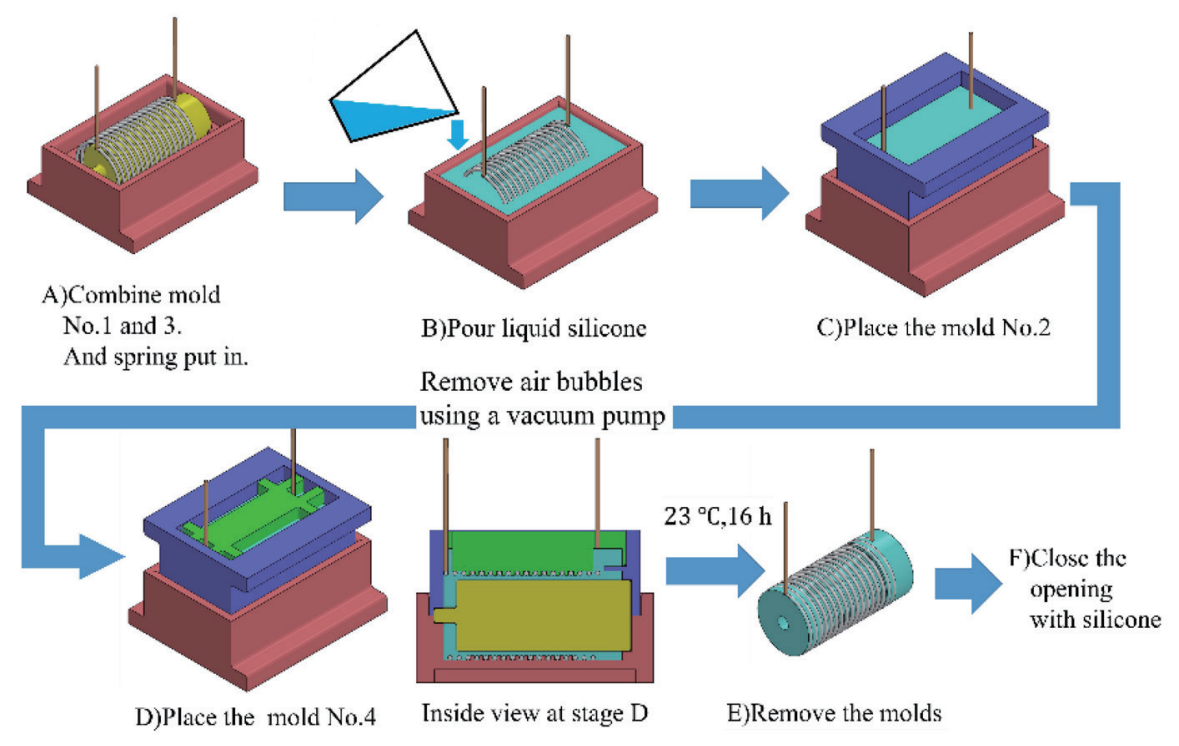

Fig. 6. (Color online) Fabrication process of the soft actuator.

acrylic plate with an air tube is bonded to the lower surface of the actuator. We do not consider the deformation of molds during the fabrication process because the deformation is small.

The repeatability of the displacement estimation was confirmed by measuring the oscillation frequency when the actuator returned to its initial length after operation. We measured the frequency eight times with the old actuator and the newly developed soft actuator. The variance for the old actuator was $6.02 \mathrm{KHz}^{2}$ and that for the newly developed actuator was $1.54 \mathrm{KHz}^{2}$. We thus confirmed the high repeatability of the new actuator.

\subsection{Specifications of new actuator}

Figure 7 shows a photograph and typical dimensions of the newly designed soft actuator. The parameters of the new actuator are as follows: initial length: $40 \mathrm{~mm}$, maximum outer diameter: $20 \mathrm{~mm}$, chamber inner diameter: $16 \mathrm{~mm}$. Both ends of the metal spring are embedded in the silicone tube, and other parts of the spring are exposed outside of the silicone as shown in Fig. 7. The weight is $13.0 \mathrm{~g}$ for the silicone part and spring alone, and $19.0 \mathrm{~g}$ including the parts for mounting the experimental equipment. This actuator extends to about 1.8 times its initial length by applying $200 \mathrm{kPa}$. By using a noncontact spring, the frequency can be stably measured at the initial position of the actuator at atmospheric pressure.

\section{Experiments}

\subsection{Experimental setup}

The experimental apparatus is shown in Fig. 8. The actuator was connected to a linear guide to constrain its movement to be parallel to a wire encoder. The actuator displacement was 


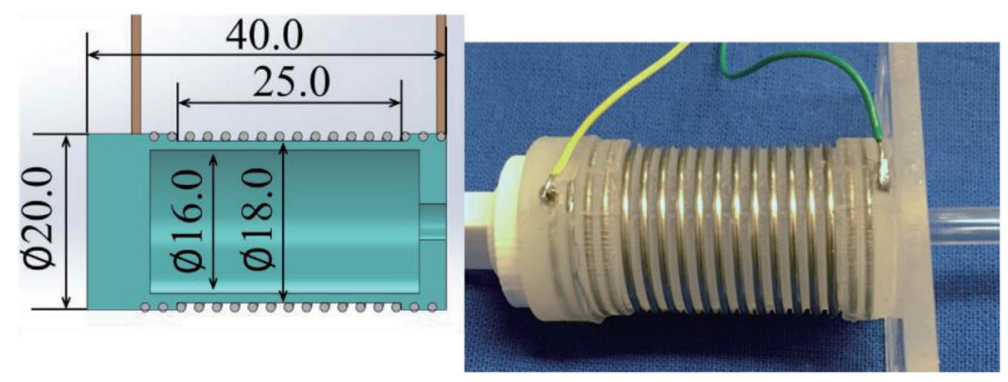

Fig. 7. (Color online) Newly designed actuator.

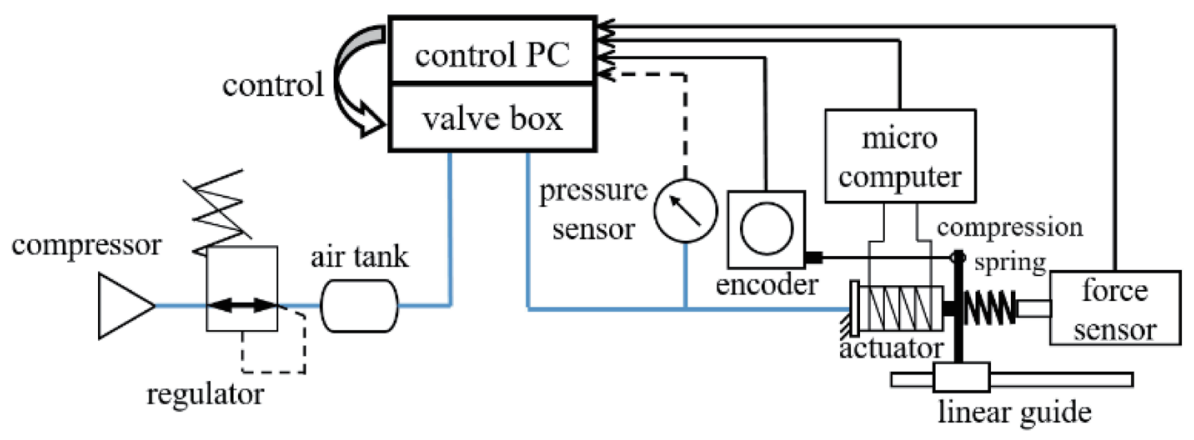

Fig. 8. (Color online) Experimental apparatus used for force measurement.

measured by the wire encoder with a resolution of $0.04 \mathrm{~mm}$. By installing the spring between the actuator and the force sensor, an external force was applied that depended on the displacement. Details of the devices used in the experiment are shown in Table 2. The specifications of the wire encoder, force sensor, servo valve, and compression spring are summarized in Tables 3-6, respectively. Experiments were conducted to confirm the accuracy of force estimation with the proposed soft actuator. The actuator was driven by pressure control and pushed the force sensor. The measured and estimated values during the driving of the proposed actuator were compared. We used a PJRC Teensy 3.2 microcomputer and the FreqCount library for oscillation measurement. This library is suitable for $1 \mathrm{kHz}-8 \mathrm{MHz}$. The oscillation pulses were counted during the gate interval by the FreqCount program, and a low-pass filter with a cutoff frequency of $50 \mathrm{~Hz}$ was applied. The operating frequency of the Teensy3.2 was $72 \mathrm{MHz}$, the data transfer rate of the serial communication was $56000 \mathrm{bps}$, and the frequency sampling time was $0.001 \mathrm{~s}$. The gate interval was $1 \mathrm{~ms}$ and the resolution of the counter was $1 \mathrm{~Hz}$ in this study.

\subsection{Acting force estimation experiments}

The actuator was operated by proportional-integral (PI) pressure control using the servo valve. The block diagram of PI control is shown in Fig. 9. The control gains were determined experimentally. The proportional gain $K_{a p}$ and the integral gain $K_{a i}$ were set to $0.04 \mathrm{~V} / \mathrm{Pa}$ and $5.5 \mathrm{~V} /(\mathrm{Pa} \cdot \mathrm{s})$, respectively. The soft actuator extended from 0 to $20 \mathrm{~mm}$ with no load at a supply 
Table 2

Experimental devices.

\begin{tabular}{lc}
\hline Servo valve & FESTO MYPE-5-M5-010B-30L-SA \\
\hline Pressure sensor & SMC PSE510-M5 \\
\hline Encoder & MLS-12-1500PST16E-250 \\
\hline Force sensor & IMADA DS2-50N \\
\hline Control PC & Ubuntu real-time OS \\
\hline AD board & 16 bit \\
\hline DA board & 16 bit \\
\hline
\end{tabular}

Table 3

Specifications of encoder.

\begin{tabular}{lc}
\hline Range of measurement & $250 \times 10^{-3} \mathrm{~m}$ \\
\hline Detection method & Incremental method \\
\hline Number of output pulses & 1500 \\
\hline Minimum resolution & $0.04 \times 10^{-3} \mathrm{~m}$ \\
\hline Highest response frequency & $50 \times 10^{3} \mathrm{~Hz}$ \\
\hline
\end{tabular}

Table 4

Specifications of force sensor.

\begin{tabular}{lc}
\hline Range of measurement & $50 \mathrm{~N}$ \\
\hline Minimum resolution & $0.01 \mathrm{~N}$ \\
\hline Sampling frequency & $0.001 \mathrm{~s}$ \\
\hline
\end{tabular}

Table 5

Specification of servo valve.

\begin{tabular}{lc}
\hline Orifice diameter & $2 \mathrm{~mm}$ \\
\hline Range of working pressure & $1 \times 10^{6} \mathrm{~Pa}$ \\
\hline Standard flow rate & $0-100 \mathrm{l} / \mathrm{min}$ \\
\hline Maximum working frequency & $115 \mathrm{~Hz}$ \\
\hline Range of working voltage & $0-10 \mathrm{~V}$ \\
\hline
\end{tabular}

Table 6

Specifications of compression spring.

\begin{tabular}{lc}
\hline Outer diameter & $12 \mathrm{~mm}$ \\
\hline Spring constant & $1.765 \mathrm{~N} / \mathrm{mm}$ \\
\hline Wire diameter & $1.2 \mathrm{~mm}$ \\
\hline Free height & $32 \mathrm{~mm}$ \\
\hline Contact height & $12 \mathrm{~mm}$ \\
\hline
\end{tabular}

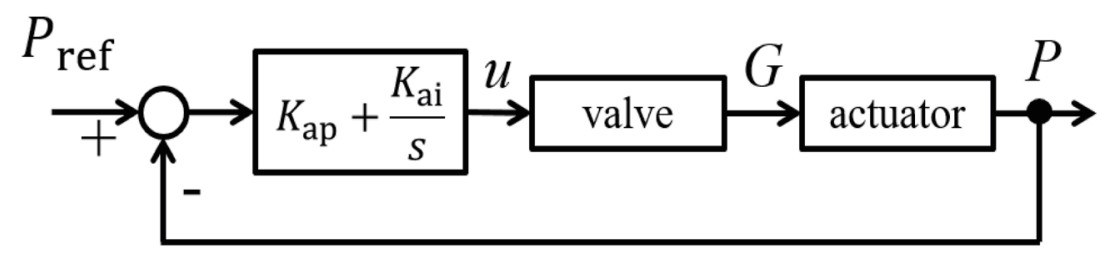

Fig. 9. Block diagram of PI pressure control.

pressure from 0 to $100 \mathrm{kPa}$. The $20 \mathrm{~mm}$ stroke was about half of the full stroke. We examined the force acting on the soft actuator in the pressure range. The soft actuator is expected to be used in low-response situations such as low-speed walking. Therefore, the input frequency of the acting force was set to $0.1 \mathrm{~Hz}$. The experiments were conducted under the following four pressure ranges to observe the effect of the hysteresis characteristics of the actuator shown in Fig. 4.

1. $0-100 \mathrm{kPa}$

2. $50-100 \mathrm{kPa}$

3. $0-80 \mathrm{kPa}$

4. $40-80 \mathrm{kPa}$

The experimental results are shown in Fig. 10. The left figures show the displacement and the right figures show the force. The blue dashed lines in the displacement figures represent 

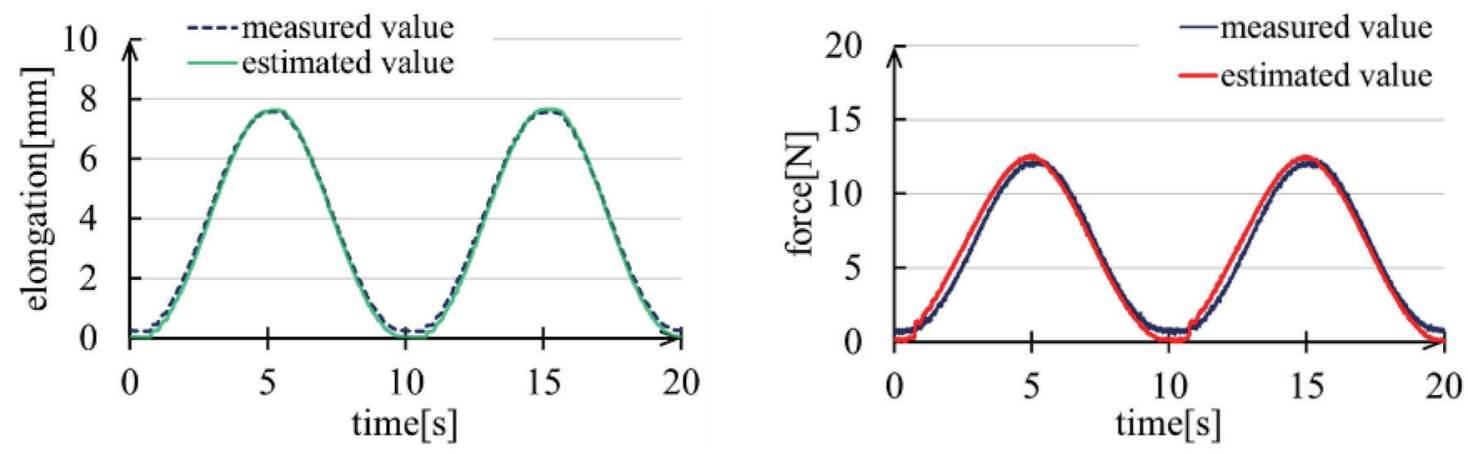

(a)
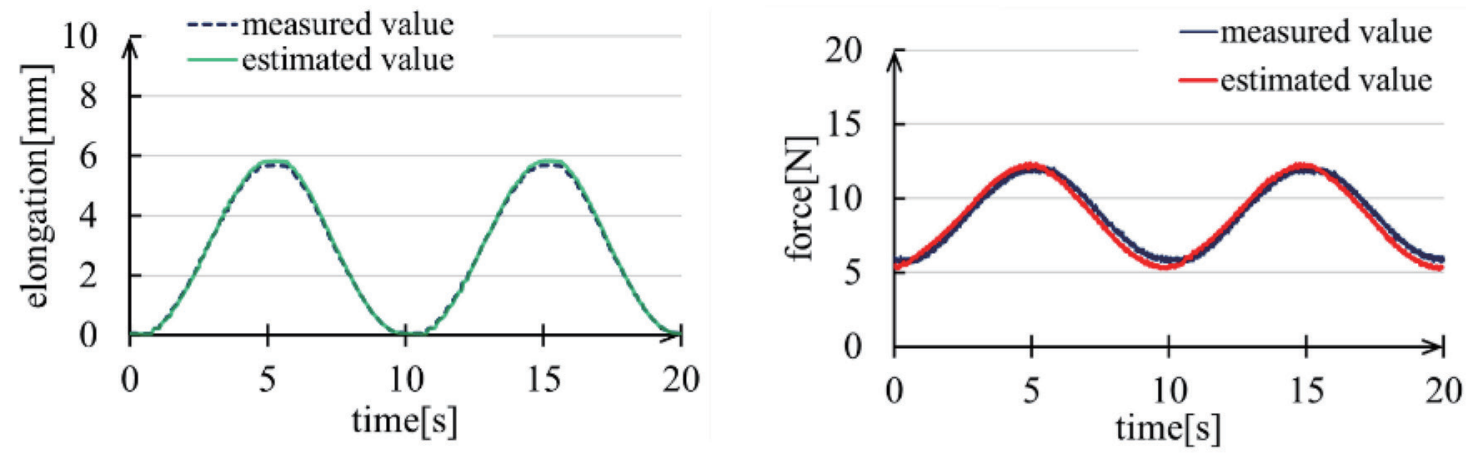

(b)
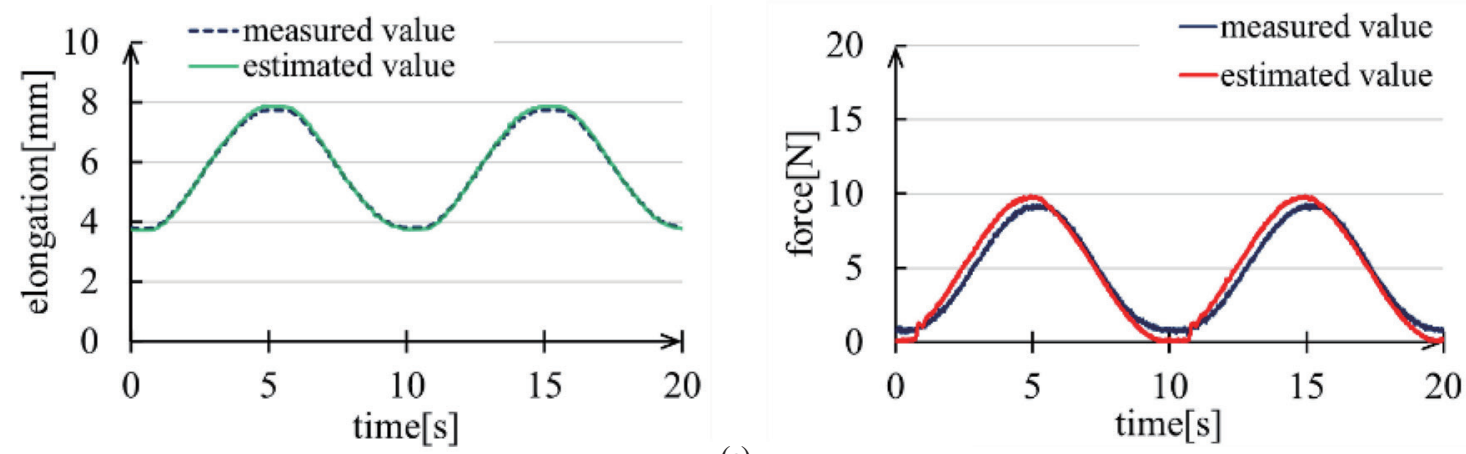

(c)
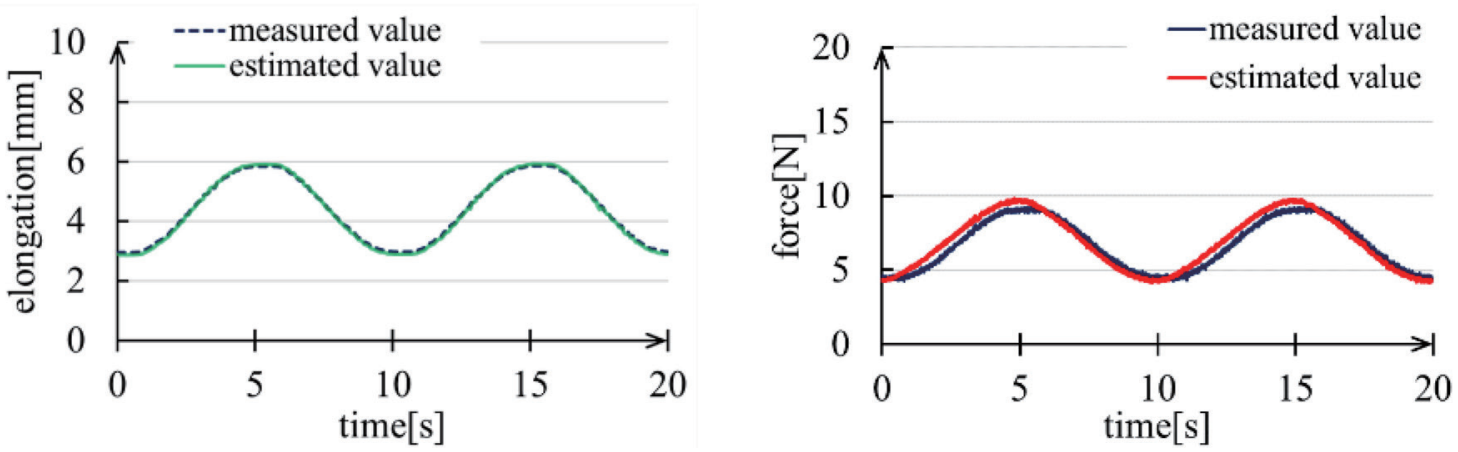

(d)

Fig. 10. (Color online) Experimental results (left: displacement, right: force). (a) Condition 1: 0-100 kPa. (b) Condition 2: 50-100 kPa. (c) Condition 3: 0-80 kPa. (d) Condition 4: 40-80 kPa. 
values measured using the wire encoder, and the green solid lines represent the values estimated from the oscillation frequency. The solid blue lines in the force figures represent the values measured using the force sensor and the solid red lines represent the values estimated using Eq. (4).

From the left figures of Fig. 10, we confirmed that the estimated displacement is in good agreement with the values measured using the encoder. From the right figures, we confirmed that the estimated force is also generally in good agreement with the values measured with the force sensor. We can see a slight phase lead in the estimated force compared with the measured force. We consider that there are two main reasons for the phase lead. First, the hysteresis characteristic shown in Fig. 4 was not taken into consideration in the force estimation. Second, the viscosity of the soft actuator was not considered since the motion is very slow. The accuracy of the estimation could be improved by considering these two factors, which will be our future work.

\section{Conclusion}

In this study, we newly designed and developed a soft actuator that fixes a spring and a silicone tube to perform displacement estimation with high repeatability. By utilizing the high backdrivability of the actuator, we estimated the force acting on the actuator from the supply pressure and the displacement. The accuracy of displacement and force estimation was confirmed by performing experiments with a low-frequency sinusoidal input of $0.1 \mathrm{~Hz}$.

There was a slight difference between the estimated and measured forces. Our future work is to improve the accuracy of the estimation by considering the hysteresis characteristics and the viscosity of the actuator.

\section{References}

1 D. Rus and M. Tolley: Nature 521 (2015) 467. https://doi.org/10.1038/nature14543

2 J. R. Amend, E. Brown, N. Rodenberg, H. M. Jaeger, and H. Lipson: IEEE Trans. Rob. 28 (2012) 341. https:// doi.org/10.1109/TRO.2011.2171093

3 X. Chen, J. Yi, J. Li, J. Zhou, and Z. Wang: IEEE Rob. Automation Lett. 3 (2018) 3505. https://doi.org/10.1109/ LRA.2018.2854409

4 Huu Minh Le, Thanh Nho Do,and Soo Jay Phee: Sens. Actuators, A 247 (2016). 323. https://doi.org/10.1016/ j.sna.2016.06.010

5 H. K. Yap, Jeong Hoon Lim, F. Nasrallah, J. C. H. Goh, and R. C. H. Yeow: 2015 IEEE Int. Conf. Robotics and Automation (IEEE, 2015) 4967-4972.

6 S. Ghate, G. Kulikovskis, and V. Shanmuganathan: 2017 Int. Conf. Data Management, Analytics and Innovation (S4DS 2017) 132-137.

7 J. Guo, Y. Sun, X. Liang, J. H. Low, Y. R. Wong, V. S. C. Tay, and C. H. Yeow: 2017 IEEE Int. Conf. Mechatronics and Automation (2017 IEEE) 1069-1074.

8 G. Feng and S. Yen: 2015 Transducers - 2015 18th Int. Conf. Solid-State Sensors, Actuators and Microsystems (IEEE, 2015) 1877-1880.

9 G. Feng and W. Huang: 2013 Transducers \& Eurosensors XXVII: The 17th Int. Conf. Solid-State Sensors, Actuators and Microsystems. (IEEE, 2013) 430-433.

10 H. Li, K. Kawashima, K. Tadano, S. Ganguly, and S. Nakano: IEEE/ASME Trans. Mechatron. 18 (2013) 74. https://doi.org/10.1109/TMECH.2011.2163415

11 E. W. Hawkes, D. L. Christensen, and A. M. Okamura: 2016 IEEE Int. Conf. Robotics and Automation (IEEE, 2016) 4022-4029.

12 E. H. Skorina, M. Luo, S. Ozel, F. Chen, W. Tao, and C. D. Onal: 2015 IEEE Int. Conf. Robotics and Automation (IEEE, 2015) 2544-2549. 
13 N. Ali, R. I. Shakoor, and M. M. Hassan: 2011 IEEE 14th Int. Multitopic Conf. (IEEE, 2011) 201-206.

14 P. H. Nguyen, S. Sridar, W. Zhang, and P. Polygerinosl: Int. J. Intell. Robot. Appl. 1 (2017) 342. https://doi. org $/ 10.1007 / \mathrm{s} 41315-017-0020-\mathrm{Z}$

15 M. C. Yuen, R. Kramer-Bottiglio, and J. Paik: 2018 IEEE Int. Conf. Soft Robotics (IEEE, 2018) 202-207.

16 N. Farrow, N. Correll: 2015 IEEE/RSJ Int. Conf. Intelligent Robots and Systems (IEEE, 2015) 2317-2323.

17 S. Trabia, V. Palmre, and K. J. Kim: 2015 12th Int. Conf. Ubiquitous Robots and Ambient Intelligence (KROS 2015) 368-369.

18 J. Morrow, H. S. Shin, C. Phillips-Grafflin, S. H. Jang, J. Torrey, R. Larkins, W. Dang, Y. L. Park, and D. Berenson: 2016 IEEE Int. Conf. Robotics and Automation (IEEE, 2016) 5024-5031.

19 Y. L. Park, B. Chen, N. O. Pérez-Arancibia, D. Young, L. Stirling, R. J Wood, E. C Goldfield, and R. Nagpal: Bioinspiration Biomimetics 9 (2014) 016007. https://doi.org/10.1088/1748-3182/9/1/016007

20 A. Koivikko, E. S. Raei, V. Sariola, M. Mosallaei, and M. Mantysalo: 2017 IEEE SENSORS (IEEE, 2017) 1-3.

21 T. Takizawa, T. Kanno, R. Miyazaki, K. Tadano, and K. Kawashima: Sens. Actuators, A 271 (2018) 124. https://doi.org/10.1016/j.sna.2018.01.007

22 R.F. Shepherd, F. Ilievski, W. Choi, S. A. Morin, A. A. Stokes, A. D. Mazzeo, X. Chen, M, Wang, and G. M. Whitesides: PNAS. 108 (2011). https://doi.org/10.1073/pnas.1116564108

23 F. Ilievski, A. D. Mazzeo, R. F. Shepherd, X. Chen, and G. M. Whitesides: Angewandte Chemie Int. Edition 50 (2011). https://doi.org/10.1002/anie.201006464

24 M. Calisti, M. Giorelli, G. Levy, B. Mazzolai, B. Hochner, C. Laschi, and P. Dario: Bioinspiration Biomimetics 6 (2011) 036002. https://doi.org/10.1088/1748-3182/6/3/036002

25 A. A. Calderón, J. C. Ugalde, J. C. Zagal, and N. O. Pérez-Arancibia: 2016 IEEE Int. Conf. Robotics and Biomimetics (IEEE, 2016) 31-38.

26 O. Azami, D. Morisaki, T. Miyazaki, T. Kanno, and K. Kawashima: Sens. Actuators, A 300 (2019). https://doi. org/10.1016/j.sna.2019.111623

27 G. S. Rao: Electromagnetic Field Theory and Transmission Lines (Wiley India, India, 2015).

28 H. Nagaoka: J. Coll. Sci. 27 (1909) 1. http://g3ynh.info/zdocs/refs/Nagaoka1909.pdf

29 T. Kanno, S. Ohkura, O. Azami, T. Miyazaki, T. Kawase, and K. Kawashima: Appl. Sci. 9 (2019) 2109. https:// doi.org/10.3390/app9102109

30 FreqCount Library: https:/www.pjrc.com/teensy/td_libs_FreqCount.html (accessed December 2020).

\section{About the Authors}

Osamu Azami received his M.S. degree in health sciences and biomedical engineering from Tokyo Medical and Dental University, Tokyo, Japan, in 2018. His research interests include soft robotics, medical robotics, and control engineering.

Toshihiro Kawase received his B.S., M.S., and Ph.D. degrees from Tokyo Institute of Technology, Tokyo, Japan, in 2007, 2009, and 2012, respectively. He was a research fellow with the Research Institute of National Rehabilitation Center for Persons with Disabilities from 2012 to 2015, and worked as a postdoctoral fellow and a specially appointed assistant professor at Tokyo Institute of Technology from 2015 to 2017. He is currently an assistant professor with Tokyo Medical and Dental University and Tokyo Institute of Technology. He is a member of IEEE. His research interests include medical robots, rehabilitation robotics, and biological signal processing. 
Tetsuro Miyazaki received his Ph.D. degree in engineering from the Department of Mechanical Sciences and Engineering, Tokyo Institute of Technology, in 2014. From 2014 to 2017, he worked as a research assistant (from 2014 to 2015) and an assistant professor (from 2015 to 2017) at Yokohama National University. From 2017 to 2020, he worked as an assistant professor at Tokyo Medical and Dental University. Since 2020, he has been an assistant professor with the Graduate School of Information Science and Technology, The University of Tokyo. He is a member of IEEE. His research interests include mechanical engineering, control engineering, power assistive devices, and medical welfare robotics.

Takahiro Kanno received his Ph.D. degree in engineering from the Department of Mechanical Engineering and Science, Kyoto University, in 2013. In 2013, he worked as a postdoctoral researcher at the Precision and Intelligence Laboratory, Tokyo Institute of Technology. From 2013 to 2019, he worked as an assistant professor at Tokyo Medical and Dental University, where he has been an associate professor since 2019. Since 2020, he has been the chief technology officer (CTO) at Riverfield Inc. He is a member of IEEE. His research interests include medical robotics, control engineering, and teleoperation.

Kenji Kawashima received his Ph.D. degree in engineering from the Department of Control Engineering, Tokyo Institute of Technology, in 1997. From 1997 to 2000, he worked as a research assistant at Tokyo Metropolitan College of Industrial Technology. He then worked as an associate professor at the Precision and Intelligence Laboratory, Tokyo Institute of Technology. From 2013 to 2020, he worked as a professor at Tokyo Medical and Dental University. Since 2020, he has been a professor at The University of Tokyo. He is a member of IEEE. His research interests include medical robotics, control engineering, and fluid measurement and control. 